\title{
URETERAL ENDOMETRIOSIS AND COEXISTENT URETHRAL LEIOMYOMA IN A POSTMENOPAUSAL WOMAN
}

\author{
ANDREW STRANG, SCOTT W. LISSON, STEVEN P. PETROU \\ Wake Forest School of Medicine, Winston Salem, North Carolina, and Department of Urology, Mayo Clinic, \\ Jacksonville, Florida, USA
}

\begin{abstract}
We report the case of a postmenopausal woman with a synchronous obstructing intrinsic endometrioma of the left ureter and a coexistent periurethral leiomyoma. Endometriosis in postmenopausal women is a rare clinical entity usually associated with exogenous estrogen use.

Urethral leiomyomas are also rare, with only 40 cases reported in the literature. Ovarian hormones are believed to influence the growth of leiomyomas. We report the genitourinary presentation of 2 separate disease entities with known hormonal influence in a postmenopausal woman receiving estrogen replacement therapy. We believe the patient's hormonal milieu affected the development of her concurrent pathology.
\end{abstract}

Key words: ureter; urethra; leiomyoma; endometriosis

Int Braz J Urol. 2004; 30: 496-8

\section{INTRODUCTION}

Endometriosis occurs rarely in postmenopausal women and is usually associated with exogenous estrogen use or excessive endogenous production by the adrenals or pituitary gland (1). Leiomyomas are benign tumors of smooth muscle origin and are infrequently found in the urinary tract, with only 40 cases of urethral leiomyoma reported in the literature $(2,3)$. The growth of both endometriomas and leiomyomas may be hormonally related. We report the case of a postmenopausal woman with a synchronous obstructing intrinsic endometrioma of the left ureter and a coexistent periurethral leiomyoma.

\section{CASE REPORT}

A 65-year-old woman was evaluated for painless gross hematuria. She denied irritative voiding symptoms or a history of urolithiasis or urinary tract infections. The patient's medications included conjugated estrogens and ramipril. Pelvic examination indicated a thickened urethra. Her laboratory test results were unremarkable. The patient had normal cystoscopic findings, and intravenous pyelography (IVP) revealed poorly opacified, dilated calices throughout the left intrarenal collecting system without visualization of the left distal ureter. Computed tomography (CT) noted a high-density mass within the left distal ureter. Ureteroscopic biopsy of the ureteral mass (Figure-1) indicated endometriosis. Magnetic resonance imaging of the urethra noted a $2.9 \times 2.9 \times 3 \mathrm{~cm}$ posterior periurethral mass (Figure-2) with signal characteristics suggestive of a leiomyoma.

The patient underwent pelvic exploratory surgery with left pelvic lymphadenectomy, left distal ureterectomy and ureteroneocystostomy. The periurethral lesion was transvaginally excised. Pathologic analysis confirmed a benign leiomyoma. 


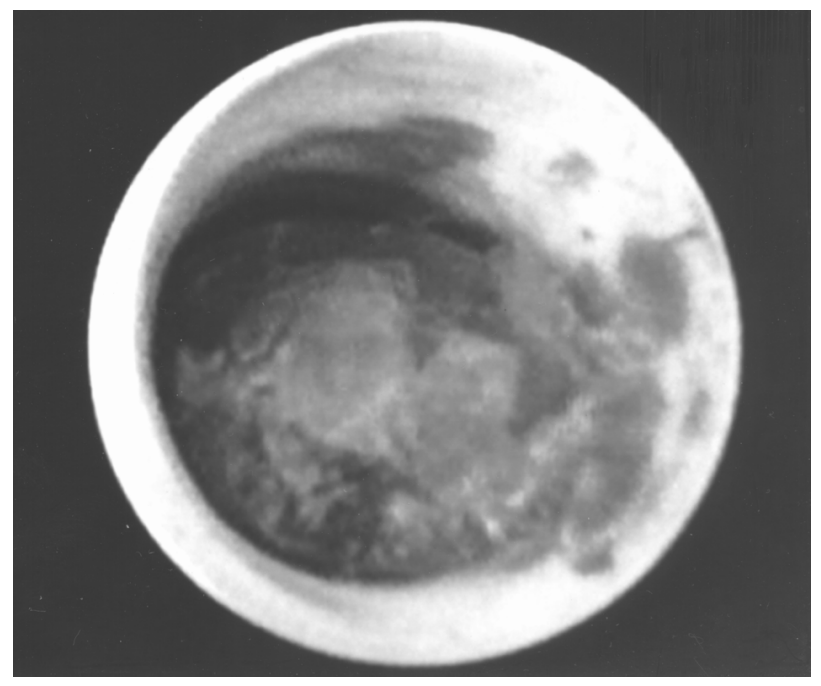

Figure 1 - Ureteroscopic image showing the ureteral lesion. Biopsy of the mass showed it to be an intrinsic endometrioma.

\section{COMMENTS}

Endometriosis in postmenopausal women is a rarity usually associated with exogenous estrogen use or excessive endogenous production by the adrenals or pituitary gland (1). Endometriosis involving the genitourinary tract has been reported with an incidence of $1.2 \%$ and a mean age between 30 and 35 years (1). The ratio of occurrence in the bladder to ureter to urethra is 40:5:1 (1). On IVP, intrinsic endometriomas tend to produce filling defects and may mimic transitional cell carcinoma and radiolucent stones. Intrinsic lesions represent advanced disease and require resection of the involved segment.

Leiomyomas are benign tumors of smooth muscle origin found rarely in the urinary tract, with 40 cases reported in the literature $(2,3)$. Leiomyomas are 3 times more common in women with an average age at presentation of 39 years (3). Common presenting symptoms include urinary tract infection, mass effect, voiding dysfunction, and dyspareunia. The most common site of urethral occurrence is the posterior wall (3). Excision is the treatment of choice.

Ovarian hormones are believed to influence the growth of leiomyomas (2). The incidence of these benign tumors increases after menarche. Estrogen and progesterone receptors have been demonstrated in

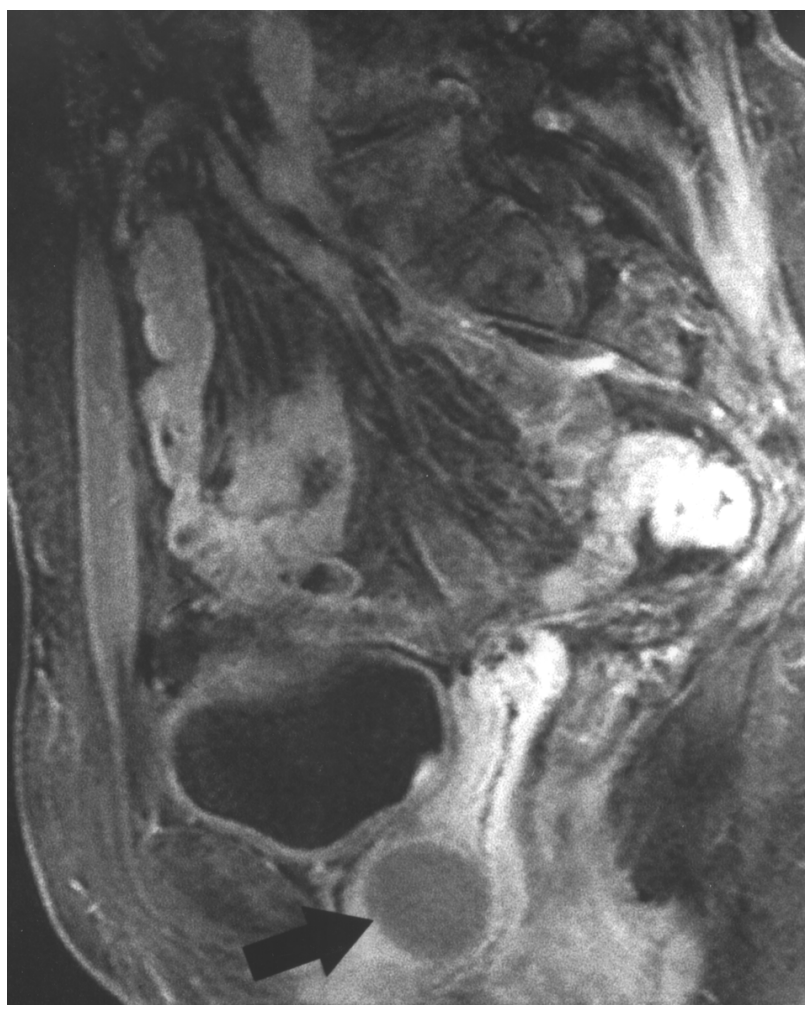

Figure 2 - Sagittal contrast-enhanced T1-weighted MRI image demonstrating a posterior periurethral mass (arrow) consistent with leiomyoma.

uterine smooth muscle tumors and underscore the rationale for medical treatment of these lesions (3). Endometriomas are also hormone-sensitive lesions with their growth likely affected by ovarian steroids. Like leiomyoma, endometriosis is treated medically with hormonal agents aimed at suppressing the hypothalamic-pituitary-gonadal production of gonadotropins.

We report the genitourinary presentation of 2 separate disease entities with known hormonal influence in a postmenopausal woman receiving estrogen replacement therapy. We entertain the likelihood that the patient's hormonal milieu affected the development of her concurrent pathology.

\section{REFERENCES}

1. Yohannes P: Ureteral endometriosis. J Urol. 2003; 170: 20-5. 
2. Alvarado-Cabrero I, Candanedo-Gonzalez F, SosaRomero A: Leiomyoma of the urethra in a Mexican woman: a rare neoplasm associated with the expression of estrogen receptors by immunohistochemistry. Arch Med Res. 2001; 32: 88-90.
3. Kurokawa S, Kojima Y, Tozawa K, Hayashi Y, Sasaki S, Kohri K: Female paraurethral leiomyoma: immunohistochemical approach to the relationship between leiomyoma and ovarian hormones. J Urol. 2002; 167: 1403-4.

Received: July 12, 2004 Accepted: August 13, 2004

\section{Correspondence address:}

Dr. Steven P. Petrou

Department of Urology, Mayo Clinic

4500 San Pablo Road

Jacksonville, Florida, 32224, USA

Phone: + 1904 953-0413

E-mail: petrou.steven@mayo.edu 